Wafa Jaroudi, Julia Garami, Sandra Garrido, Michael Hornberger, Szabolcs Keri and Ahmed A. Moustafa*

\title{
Factors underlying cognitive decline in old age and Alzheimer's disease: the role of the hippocampus
}

DOI 10.1515/revneuro-2016-0086

Received December 22, 2016; accepted February 18, 2017; previously published online April 19, 2017

\begin{abstract}
There are many factors that strongly influence the aetiology, development, and progression of cognitive decline in old age, mild cognitive impairment (MCI), and Alzheimer's disease (AD). These factors include not only different personality traits and moods but also lifestyle patterns (e.g. exercise and diet) and awareness levels that lead to cognitive decline in old age. In this review, we discuss how personality traits, mood states, and lifestyle impact brain and behaviour in older adults. Specifically, our review shows that these lifestyle and personality factors affect several brain regions, including the hippocampus, a region key for memory that is affected by cognitive decline in old age as well as AD. Accordingly, appropriate recommendations are presented in this review to assist individuals in decreasing chances of MCI, dementia, AD, and associated symptoms.
\end{abstract}

Keywords: Alzheimer's disease; cognitive decline; hippocampus; lifestyle; memory; mood states; personality.

*Corresponding author: Ahmed A. Moustafa, School of Social Sciences and Psychology, Western Sydney University, Sydney 2751, NSW, Australia, e-mail: a.moustafa@westernsydney.edu.au; and MARCS Institute for Brain, Behaviour, and Development, Western Sydney University, Sydney 2751, NSW, Australia

Wafa Jaroudi and Julia Garami: School of Social Sciences and Psychology, Western Sydney University, Sydney 2751, NSW, Australia

Sandra Garrido: MARCS Institute for Brain, Behaviour, and Development, Western Sydney University, Sydney 2751, NSW, Australia

Michael Hornberger: Norwich Medical School, University of East Anglia, Norwich NR4 7TJ, UK; and Dementia and Complexity in Later Life, NHS Norfolk and Suffolk Foundation Trust, Norwich NR4 7TJ, UK

Szabolcs Keri: NyíröGyula Hospital, National Institute of Psychiatry and Addictions, Budapest, Hungary; Department of Physiology, Faculty of Medicine, University of Szeged, 6720 Szeged, Hungary; and Department of Cognitive Science, Budapest University of Technology and Economics, 1111 Budapest, Hungary

\section{Introduction}

The occurrence of cognitive decline in old age depends on many differences among individuals. In this study, we review research addressing personality, mood, and lifestyle factors underlying cognitive decline in old age that may vary across individuals. Identifying these factors will help therapists address such factors at a younger age, thus reducing chances of developing old-age-related neurological disorders. Cognitive decline in later life commonly occurs as part of a range of neurological conditions that come under the umbrella term of dementia. Alzheimer's dementia (AD) is the most common form of dementia, with no cure existing today. It has a higher occurrence rate in females than in males (approximately 56\% and 31.4\%, respectively) and often occurs in individuals 65 years or older (Alzheimer's Association, 2014). AD is also a common cause of death in elderly individuals (National Institute on Aging, 2015). Like healthy ageing, various individual differences impact the progression of $\mathrm{AD}$, including lifestyle (e.g. type of diet and amount of exercise) and personality (e.g. neuroticism and exposure to/coping with stress).

In this review, we explore the evidence for lifestyle and personality traits that influence individuals' rate of cognitive decline in old age as well as onset of AD pathophysiology. In addition, we consider emotional states associated with sadness, stress, and loneliness, which also lead to mild cognitive impairment (MCI) and $\mathrm{AD}$ symptoms. Moreover, older adults' and AD patients' lifestyles such as diet and exercise patterns will be discussed, as well as how lifestyle can improve or worsen cognitive function and disease progression.

\section{Personality effect on cognitive decline}

\section{Neuroticism}

Personality traits such as neuroticism generally have negative impacts on normal functioning; they may be linked 
to increased risks of developing cognitive decline and dementia (Archer et al., 2009). Neuroticism is a personality trait characterised by moodiness, anger, fear, anxiety, and jealousy, among others. According to Chapman et al. (2012), older individuals scoring high on neuroticism scales but low on openness (e.g. being accepting of change) and conscientiousness (e.g. being careful, organised, and mindful) scales exhibit poor long-term cognitive functioning and steeper rates of cognitive decline (Chapman et al., 2012). A possible explanation for cognitive decline in old age is hippocampal atrophy related to glucocorticoid drugs (Sapolsky, 1996) such as chronically elevated cortisol levels (Chapman et al., 2012). Increased cortisol has been associated with stress characteristics of negative affect (Buchanan et al., 1999), which may relate to neuroticism. In addition, emotional stress and glucocorticoids (associate characteristics of neuroticism) may also lead to structural changes in the hippocampus (atrophy and volume loss), further contributing to cognitive decline (Gurvits et al., 1996; McEwen, 2000; Boyle et al., 2010). Changes in these personality domains are significantly high in $\mathrm{AD}$ patients, where premorbid personality traits have a moderate association with levels of cognitive functioning, and functioning in daily life (Chapman et al., 2012).

In females, midlife neuroticism seems to be associated with the early onset of dementia (Archer et al., 2009). In comparison to males, female midlife neuroticism may be accounted for by lower levels of the hormone oestrogen at menopause (considering that $\mathrm{AD}$ usually occurs after the mean age of 65 years). If not managed, stress can develop into distress, increasing risks of cognitive decline, impairment, and also $\mathrm{AD}$ (Wilson et al., 2007a).

Neuroticism, in comparison to other personality traits, can be suggested to present with elevated activity of the limbic brain (Newbery, 2012), which includes the hippocampus, dentate gyrus, cornuammonis, amygdala, basal ganglia, as well as other regions. The limbic system related to emotion and memory involves the amygdala and hippocampus. A correlation between activation levels in the amygdala and hippocampus (Poulin et al., 2011) suggests that atrophy in both may relate to less emotional reactivity in general and in association to memories. During mild AD, it is noted that amygdala activity is most associated with elevated emotions such as irritability and anxiety (Poulin et al., 2011), which highlights the function of brain alterations that occur in the hippocampus related to neuroticism and cognitive decline in $\mathrm{AD}$ (Boyle et al., 2010).

\section{Mood and emotional state effect on cognitive decline}

\section{Loneliness}

Prolonged feelings of loneliness can harm individuals in many ways. Loneliness can lead to depression and higher risk of death (Holwerda et al., 2016). Feeling lonely can sometimes also lead to thoughts of being a burden or a liability on family and friends (Davis, 2014). Additionally, although patients with amnesia (and probably AD) show memory decline, they still feel emotions associated with memories (University of Iowa, 2010). In an attempt to improve patient rate of cognitive decline, it could be suggested to improve patients' psychological wellbeing, including stress levels. This can include providing comfort, care, and intimacy. Care is necessary for patients to feel understood and appreciated by others, especially by family, caregivers, nurses, and friends (Anderson, 2007). Observations indicate that loneliness doubles the risks of late-life AD-like dementia (Wilson et al., 2007b); however, there have been mixed findings. Autopsies suggest that loneliness is not related to $\mathrm{AD}$ or cerebral infarction (Wilson et al., 2007b). However, we believe that although the impact of loneliness may not be directly observable, it can influence depression, stress, and similar factors that do have direct impacts on cognition. As such, it is reported that depression associated with loneliness may be a contributing factor of AD development (Tanday, 2007).

\section{Stress}

Stress and distress (including anxiety) can have many health implications. This includes increased risks and exacerbation of cognitive decline and functioning, MCI, and AD (Wilson et al., 2005, 2007a, 2011). This may be explained in part by stress-related release of the hormone cortisol, as it has been found that extreme stress leading to excessive release of cortisol affects the hippocampus (Gurvits et al., 1996; Buchanan et al., 1999; McEwen, 2000). Extreme elevated cortisol levels influence hippocampal function and result in impaired cognition, specifically memory (Archer et al., 2009). Reviewing research in the previous section on the personality trait of neuroticism and now, stress, it is notable that a high level of cortisol is common in the two. The linkage concerns increased cortisol leading to hippocampal atrophy (Chapman et al., 2012), which consequently contributes to memory 
declination (Archer et al., 2009). To reduce stress and cortisol levels and their impact on cognition, we suggest that individuals switch to a healthy diet and participate in exercise regularly as part of a healthy lifestyle.

Stress is also a significant risk factor of amnestic MCI (aMCI). In a study conducted by Katz et al. (2016), participants were assessed 3 years after initial risk assessment of aMCI. It was found that the risk of developing aMCI increased by 30\% per five-point score on the Perceived Stress Scale. Participants with scores in the 80th percentile were almost 2.5 times more likely to develop aMCI over this time.

Moreover, females often express higher rates of distress, which is associated with a decline in cognitive functioning over an average of 5 years (Wilson et al., 2005). Increased experience of stress in females may explain why worse cognitive functioning is more common in females than in males (Wilson et al., 2005).

As well as increasing the risk of cognitive decline, negative mood states and experiences involving stress, anxiety, and so on may also contribute to an acceleration of cognitive decline in people with diagnoses of various forms of dementia (Wilson et al., 2005). Chronic distress and emotional states (including misery and agony) are common in AD patients. Irritability, agitation, and similar emotions also contribute to worse $\mathrm{AD}$ patient health and may also affect cognition (Hurt et al., 2010). High levels of continual distress are associated with decline in multiple cognitive functions, primarily episodic memory (Wilson et al., 2007a). Moreover, chronic distress is also linked to higher rates of MCI. In addition to an association with global cognitive impairment, chronic distress is also related to limbic system functioning (Wilson et al., 2007a). However, chronic distress is suspected to specifically influence certain aspects of cognition more than others, predominantly episodic memory (Wilson et al., 2007a). This association between chronic distress and memory may be tied to the relationship between the hippocampus and limbic system. The anatomic structure of the limbic cortex is connected with the hippocampus; therefore, if the limbic system is altered by stress, the hippocampus may also be affected, likely to disrupt memory (Tortora and Derrickson, 2006). This reflects a chain-like link or hierarchical structure where all factors associated with cognitive decline lead or contribute to one another.

Studies of posttraumatic stress disorder (PTSD) patients may additionally help shed light on the connection between stress, distress, and cognitive decline. There are increased risks of developing dementia in male veterans under the age of 75 with PTSD (Yaffe et al., 2010), which suggests that an individual's stressful life experiences increase the likelihood of developing neurodegenerative diseases. An increased risk of disease development has been attributed to damage to hippocampal structures. Suffering from head injury, witnessing traumatic events, and experiencing chronic stress can damage or shrink the hippocampus, causing disruption to learning and memory (Yaffe et al., 2010). Stressful experiences tend to overlap with ongoing anxiety, which is very common in elderly individuals with cognitive decline and dementia (Bierman et al., 2007). As anxiety increases, so does global cognitive decline, making individuals more prone to stress (Wilson et al., 2011). Moreover, cognitive decline is related to the amount of stress an individual experiences, increasing the chances of developing AD (Wilson et al., 2011).

\section{Depressive symptoms}

Individuals experiencing depression are suggested to also score high on neuroticism (Farmer et al., 2002), highlighting the importance of considering both factors in AD. It is proposed that depression is associated with hippocampal volume loss (Sheline et al., 2003) and contributes to cognitive decline and dementia (Yaffe et al., 1999). However, much of the literature consider depression and stress and their associated emotions separately when assessing and evaluating individual's mental well-being, cognition, and risk of $\mathrm{AD}$, despite the fact that such emotional psychological states are closely related (Nima et al., 2013). We attempt to bridge this gap by discussing the mentioned states and emotions, considering their pathological symptoms, and suggesting strategies for minimising the effect of psychological distress on individuals in mid and late life.

Depression has been found to be a contributing factor to dementia and AD (Cherbuin et al., 2015). In elderly individuals' assessments of depression, each point increase on the Center for Epidemiological Studies Depression/ Hamilton Depression Scale (CES-D/HAM) contributes to a $5 \%$ increase risk of dementia over a period of 5.2 years (Cherbuin et al., 2015). Furthermore, scores above 20/21 on the CES-D and similar scales used to measure depression are linked to an $83 \%$ increase in the risk of dementia, after an 8.2-year follow-up. Similarly, a one-point increase on the CES-D/HAM was found to increase AD development by $6 \%$ over a 5.9-year period (Cherbuin et al., 2015). In more detail, Cherbuin et al. (2015) also note that scores above 20/21 on the CES-D is linked to a $97 \%$ risk increase of AD over 6.3 years. These findings are also where dementia 
patients tend to score high on the CES-D (Fuhrer et al., 2003), implying that depression is related to cognitive decline and dementia development.

Depression is suggested to also relate to pathological issues such as neuroinflammation, oxidative stress, white matter lesions, and cerebral atrophy (Cherbuin et al., 2015). Such pathological issues are evident even in young individuals with depression. Therefore, for practitioners to minimise chances of developing dementia and $\mathrm{AD}$ at a young age, it would be best to consider the cause, treatment, and management of pathological issues linked with the development of depression. In fact, it has been argued that a $25 \%$ reduction in depression prevalence could result in 827000 fewer cases of AD worldwide (Barnes and Yeffe, 2011). While managing depression, it should be noted that verbal types of therapy may not be across the board effective because individuals respond differently to forms of therapy. For example, elderly and young males are less likely to engage in emotion talk therapy or even seek support from such therapies (Anderson and Brownlie, 2011). Therefore, it may be more beneficial for individuals to seek support from a wider range of therapies. For instance, in mild and moderate dementia, music therapy not only assists individuals to cope with and reduce depressive symptoms but also improves cognitive functioning and processes (mainly recall) in a positive and effective manner (Chu et al., 2014).

Apathy, an affective state involving a lack of interest, also affects cognition. Although apathy and depression are different, they can co-occur but are reliant on different neural mechanisms. As mentioned previously, it is important to account for factors such as depression and apathy as occurring if not combined, then hierarchically in dementia development. Depression and apathy may relate to each other on the basis of similar emotional affects (Benoit et al., 2012). This is supported by the finding that depressed individuals tend to exhibit apathy, and individuals with apathy tend also to show depressive symptoms (Benoit et al., 2012). Therefore, we could suggest a unique symptom representative of the overlap between depression and apathy (Benoit et al., 2012) in cognitive decline. Regarding neuropathological effects of apathy, highly apathetic patients develop cortical emaciation in the caudal anterior cingulated cortex, lateral orbitofrontal cortex, and pars triangularis where neurofibrillary tangles occur (Tunnard et al., 2011). Changes in these brain regions are suggested to contribute to cortical thinning of the left anterior cingulate, affecting memory among other cognitive processes (Tunnard et al., 2011). Therefore, it is hypothesised that the underlying psychopathological mechanism connecting apathy and depression with cognition is the role of the anterior cingulated cortex (ACC). It could be suggested that damage to the ACC affects emotional experience and expression, as well as cognitive functions like learning.

Awareness of one's disease (AD), also known as insight, has been associated with depression, anxiety, and apathy (Horning et al., 2014). Insight is defined as one's self-understanding, knowledge, and awareness of cognitive decline, impairment, and disease diagnosis. On the other hand, anosognosia is the lack of knowing and understanding one's own cognitive and functional difficulties. In elderly individuals and AD patients, anosognosia can limit some abilities, leading to risky and harmful behaviour (e.g. unknowingly overdosing on medications or accidentally keeping the stove on high heat, causing a fire) (Starkstein et al., 2007). Risky behaviour may be related to the idea that $\mathrm{AD}$ patients experience problems with verbal comprehension, fluency, and constructional praxis, which may be linked to completing certain processes and functions in a proper and safe manner (Starkstein et al., 2007). Impairment in aspects of cognition is influenced by patients' awareness and knowledge of their physical and mental health. Lack of self-knowledge continues to degrade with cognitive functioning, as supported by Mini-Mental State Examination (MMSE) scores (Hurt et al., 2010). With continued lack of insight and cognition, anxiety levels start to decrease because anxiety invoking experiences start to have no significant emotional effect (Bierman et al., 2007).

\section{Distinguishing cognitive decline from typical mood state symptoms}

Many individuals in mid and late life exhibit neuroticism and experience loneliness or stress. However, it is imperative to distinguish between symptoms inherent to aging and those indicative of reversible or solvable loneliness, stress, and depression. In doing so, it is vital to consider both observable behaviour and neurological changes. For example, clinicians often ask if patients are feeling depressed and if they complain of memory problems. If depressed, it is likely that they do not have dementia, as depression has a major impact on episodic memory functioning.

When investigating the differences between physical symptoms of mood changes and age-related cognitive decline, it is important to monitor amygdala activity and hippocampal changes. This includes observing expressions of irritability and anxiety (Poulin et al., 2011) as 
indicative of possible signs of the cognitive aging process. It may be possible that if all factors of amygdala activity, hippocampal changes, and expressions of irritability and anxiety occur together or are subsequent to each other, individuals may be in the early stages of behavioural and cognitive decline. If this is found not to be the case, it is essential to consider other factors such as environment. For example, individuals' living spaces are another important factor in cognition as a lonely environment may contribute to global cognitive impairment (O'Luanaigh et al., 2012). It is also important to not only note individuals' emotional experiences of loneliness but also test memory loss (University of Iowa, 2010) and assess individuals for stress and depression, and compare scale scores to that of MMSE scores (Wilson et al., 2005; Katz et al., 2016).

An additional approach to distinguishing temporary reversible emotional states and moods from those that are age induced is to consider the crucial aspects of cortisol levels and brain alterations, such as increased risk of dementia, atrophy, and lesions to regions of the limbic system such as the hippocampus, amygdala, and ACC (Tunnard et al., 2011; Chapman et al., 2012). Such assessments will assist researchers and therapists to identify whether an individual is experiencing cognitive impairment that is induced by reversible, temporary, or environmental factors (pseudo-dementia) rather than confusing it with other types of dementia (AD).

\section{Lifestyle factors in cognitive decline}

\section{Nutrition}

Diet has been found to be related to cognitive decline (Nurk et al., 2007). There is evidence that tea, fish, fruits, nuts, and turmeric (curcumin) may protect against dementia (Nurk et al., 2007; Mishra and Palanivelu, 2008; Chen et al., 2011; Pe'neau et al., 2011; Poulose et al., 2014; Qin et al., 2014; Cardoso et al., 2016). It is suggested that vitamins, proteins, and amino acids may explain their neuroprotective effects (Ng et al., 2008; Joseph et al., 2009; Xu et al., 2009; Rest et al., 2013). Below, we discuss the benefits of some foods associated with improved cognition and physical health.

Tea is understood to have overall health benefits, including that of reduced cognitive decline and improved MCI (Ng et al., 2008; Park et al., 2011). Green tea is found to be associated with lower rates of cognitive impairment (Kuriyama et al., 2006; Ng et al., 2008), and black fermented and semifermented tea (oolong) have both been shown to significantly reduce cognitive decline in old age (Ng et al., 2008). This is possibly due to naturally occurring chemical compounds such as theanine in both black and green tea (Ng et al., 2008; Park et al., 2011). Natural compounds found in various types of tea reduce acetylcholinesterase activity and protect $\beta$-amyloid driven affects on cognition (Ng et al., 2008).

Fish may not only improve cognition but may also act as a neuroprotective agent against AD. Elderly individuals who consume fish or seafood weekly show a delay from 4 to 7 years of developing dementia and AD (Nurk et al., 2007). Consuming more than one serving (e.g. $100 \mathrm{~g}$ ) of fresh water fish is associated with 2.5 times slower cognitive decline in verbal, immediate, and delayed memory (Qin et al., 2014). Potential explanations of these findings may be omega-3 polyunsaturated fatty acids (w-3 PUFA), eicosapentaenoic acid (EPA), and docosahexaenoic acid (DHA) concentrations in fish, which have been shown to reduce inflammation and oxidative stress in the central nervous system (Qin et al., 2014). However, it is suggested that reduction of inflammation and stress prevents cognitive decline but does not prevent or treat dementia (Qin et al., 2014). Nurk et al. (2007) also identified that both unprocessed fatty and lean fish lead to significant improvements in overall cognitive performance. However, it is suggested that consumption of fatty fish has more protective effects against dementia and $\mathrm{AD}$ than does consumption of lean fish (Huang et al., 2005), which may be related to the nutritional factors found in fatty fish such as higher DHA and PUFA (Zhang et al., 2016). Moreover, processed fish is noted to be the second most common contributor to improving episodic and short-term memory, visuospatial and motor skills, naming and recall, among other cognitive functions (Nurk et al., 2007). Enhanced cognition has also been associated with the consumption of fish oil (O'Connor et al., 2012), which is apparent in semantic memory, verbal fluency, and psychomotor speed (Nurk et al., 2007).

Various nutrients have been found to affect cognition differently. Vitamins C and E contained in fruits such as strawberries and blueberries have shown to improve verbal memory in middle- to old-aged individuals (Pe'neau et al., 2011). Moderate fruit consumption is positively correlated with cognition in individuals with MCI (Chen et al., 2011). Antioxidants and polyphenolics present in some fruits (e.g. concord and blueberry juice) are thought to mediate improvements in cognitive functions such as memory by contributing to the reversal of aging neurons and forgetful behaviours typically observed in elderly individuals (Joseph et al., 2009).

Nuts have many benefits, including that of improving cognitive function (e.g. reducing memory decline) (Nooyens 
et al., 2011). Specifically in relation to walnuts, they are suggested to reduce chances of $\mathrm{AD}$ by managing $\mathrm{A} \beta$ peptides and protecting cells from toxicity of free damaging radicals with its antioxidant properties (Muthaiyah et al., 2011). Walnuts, rich in polyphenol and melatonin when taken in moderate amounts, improve cognition (memory) and motor function (Willis et al., 2009), possibly because polyphenols reduce oxidative stress and inflammation (Poulose et al., 2014). Walnuts also have a high content of PUFAs (EPA and DHA), found to improve and maintain cell and nerve functioning (Yehuda et al., 2002). PUFAs enhance synaptic plasticity and stability of the neural membrane, which contributes to neural transmission, and also play a role in gene expression and neurogenesis (Yehuda et al., 2002). Also found in walnuts is the amino acid tryptophan, which may improve cognitive functioning of elderly individuals. Tryptophan produces serotonin, improving aspects of verbal and short-term memory by influencing effective transmission of neural signals and messages to and from the brain (Rest et al., 2013). In Brazilian nuts, the mineral selenium is also suggested to be beneficial in the prevention of dementia. With its antioxidant properties, selenium protects the body from free radicals, oxidative stress, and other neuropatholgoical complexities that negatively influence neurotransmission (Cardoso et al., 2016). Brazilian nuts are found to reduce and restore selenium in elderly individuals, improving semantic verbal fluency and constructional praxis among other less focused cognitive functions (Cardoso et al., 2016).

Turmeric (curcumin) is a natural anti-inflammatory plant that is used as a spice and natural herbal medicine. Curcumin has been shown to reduce inflammation commonly found in neurodegenerative diseases such as $\mathrm{AD}$ (Mishra and Palanivelu, 2008). Additionally, curcumin can reduce infarction, deficit, and oxidative stress and prevent excessive formation of amyloid plaques (Mishra and Palanivelu, 2008). Curcumin has effects on proteins and cells such as cytokines and macrophages, which have a crucial role in internal bodily responses against amyloid plaques that occur in AD (Xu et al., 2009). Curcumin also has beneficial effects similar to that of omega-3 fats in that it reduces inflammation and oxidative stress affecting the brain (Xu et al., 2009).

\section{Fitness and exercise}

It is suggested that physical activity reduces cognitive decline associated with AD (Larson, 2008). Fitness influences mental and physical well-being and is associated with improved cognition. Regular exercise contributes to protecting individuals from experiencing hippocampal volume loss, brain atrophy, and declined memory, which are characteristics of AD (Erickson et al., 2011). Young individuals with high fitness levels and who regularly participate in cardiorespiratory fitness are twice less likely to develop dementia, including $\mathrm{AD}$ (Liu et al., 2011). Similar findings were found in healthy individuals without dementia, in that individuals with increased rates of daily physical activity had significantly reduced risks of AD over 4 years (Buchman et al., 2012). Data analyses also showed a negative correlation between physical activity and decline in episodic, working memory and visuospatial cognitive functions (Buchman et al., 2012).

Furthermore, moderate participation in fitness, in general, specifically aerobics, protects from and reduces old-aged hippocampal volume loss (Erickson et al., 2011). Aerobic exercise is found to reverse volume loss and increase volume of the anterior hippocampus (Erickson et al., 2011). In addition, an increase in amount of grey and white matter of the prefrontal cortex is apparent in exercise participation (Erickson et al., 2011). Exercise is also assumed to be associated with increases in brain-derived neurotrophic factor serum levels and enhancements to anterior hippocampal volume, which in turn improves memory (Erickson et al., 2011). Thus, it can be suggested that exercise improves neuronal growth and cerebral blood flow, increases brain volume, and improves learning and memory (Sullivan, 2008; Erickson et al., 2011). High levels of fitness in $\mathrm{AD}$ patients are also related to a four times reduction in brain atrophy than individuals with low levels of fitness. Exercise also contributes to increased white matter volume in the hippocampal, inferior temporal gyrus, and precentralgyrus brain regions (Sullivan, 2008).

In sum, involvement in physical activity such as aerobics may improve executive control and train regions of the brain such as the hippocampus. This may relate to exercise increasing dentate gyrus cerebral blood volume, which improves cognition (learning) (Pereira et al., 2007). The benefits of fitness are important to advocate in order to reduce young individuals' risks of cognitive and functional impairment in later life.

\section{Sleep}

Low hours of sleep at night are also associated with lack of exercise (Ohayon and Vecchierini, 2005), which may result 
in having fewer protections against cognitive decline. Also related is the idea that neurogenesis in individuals who exercise is the correlation of oxygen volume used by individuals (Pereira et al., 2007), suggested to take part in neurogenesis, which sleep dysfunction may interrupt, leading to cognitive decline. In support of this idea is that sleep disturbance and fewer hours of sleep have also been shown to be risk factors for cognitive decline, dementia, and AD development (Osorio et al., 2011; Spira et al., 2014). Ohayon and Vecchierini (2005) reported obstructive sleep apnoea (OSA), rapid eye movement (REM), and other sleep factors as contributing to cognitive decline.

Presenting a potential relationship between OSA and cognitive processing is Stranks and Crowe (2016) suggesting impaired patients presenting worse executive function (Alchanatis et al., 2004; Ancoli-Israel et al., 2008), specifically nonverbal memory, concept formation, and other functionality and psychomotor speed (Stranks and Crowe, 2016). Individuals who received intervention for OSA using nasal continuous positive airway pressure (CAP) have shown improved cognition in aspects of memory and executive function, which is suggested to be affected by interruption of breathing during sleep (Ancoli-Israel et al., 2008). The way in which OSA may impact on cognition includes lack of oxygen reaching brain tissue (hypoxia), leading to brain cell injury, associated with individuals' bodily functioning experiencing a state of oxygen deficiency (anaerobic glycolysis) (Alchanatis et al., 2004). Although CAP apnoea machines can support breathing (oxygen intake) to lessen the chances of cognitive impairment, they cannot completely repair damage caused by OSA or make up for the benefits of normal, healthy sleep function (Alchanatis et al., 2004; Ancoli-Israel et al., 2008), which may involve the role of neurogenesis.

Another sleep factor shown to affect cognition is REM sleep (Ohayon and Vecchierini, 2005). Some studies show how REM sleep may be related to cognitive impairment in elderly individuals and in relation to emotional and mood states. In middle to elderly aged men, decreased time in the REM stage of sleep was associated with elevated cortisol during evening time of the day, contributing to memory decline (Van Cauter et al., 2000). It has also been reported that individuals with MCI are likely to develop REM sleep behavioural disorder (RBD) (Gagnon et al., 2012). The signs of cognitive impairment upon developing RBD involve memory, attention, and concentration dysfunction, among many other symptoms, which commonly occur in neurodegenerative diseases such as AD, Parkinson's disease, and Lewy Bodies dementia (Gagnon et al., 2012).

\section{Conclusion}

Factors associated with cognitive decline in elderly individuals include personality, mood, and lifestyle. Personality and mood states either negatively or positively influence memory performance, most likely by affecting hippocampus function. The extent that factors contribute to risks of cognitive decline usually determines the delay or onset of neurodegenerative diseases such as dementia and AD. Making appropriate and necessary lifestyle changes may minimize the risks and severity of cognitive decline and impairment.

In response to factors contributing to cognitive decline, further considerations need to be made and studied in the future. The findings of Katz et al. (2016) emphasise the importance of considering different aspects of individuals' living environment. Creating a positive environment, especially for the elderly, can lessen the chances of experiencing sadness, stress, anxiety, and similar emotions associated with cognitive decline, aMCI, $\mathrm{AD}$, and other age-related diseases. Some necessary lifestyle changes include nutrition, fitness, and the care and company of family, friends, and caregivers, which can contribute to creating a positive and socially rich environment for elderly individuals. The significance of such implications involves lifestyle and environmental changes that not only influence mood states, personalities, symptoms, and disease progression but are also associated with brain changes.

Other factors contributing to cognitive decline that can be investigated in the future regard individual differences in terms of education and language quality. One interesting aspect is the association of individuals' level of education and age-related neurodegenerative diseases (Katz et al., 2016). Individuals who tend to be highly educated and use sophisticated vocabulary, present good language expression of thoughts and ideas, and have complex sentence structure tend to develop dementia at a later age than usual (Marx, 2005). On the other hand, those with poor language qualities suggesting low education levels indicate that education may be beneficial and exercise cognition, reducing risks of $\mathrm{AD}$, thus making education and language future interests to explore.

An additional recommendation for future work involves the study of the complex properties of languages. As a main form of communication, languages with different characteristics may influence cognitive reserve, decline and impairment, dementia, and AD. It is also important to note that how and when language 
abilities are developed influence levels of language comprehension, processing, and use (Bialystok and Luk, 2012), which, in turn, might affect levels of cognition, cognitive reserve, and control.

Acknowledgments: S.G. is funded by an ARC/NHMRC Dementia Research Development grant. M.H. is funded by Alzheimer's Research UK and the Wellcome Trust.

\section{References}

Alchanatis, M., Deligiorgis, N., Zias, N., Amfilochiou, A., Gotsis, E., Karakatsani, A., and Papadimitriou, A. (2004). Frontal brain lobe impairment in obstructive sleep apnoea: a proton MR spectroscopy study. Eur. Respir. J. 24, 980-986.

Alzheimer's Association (2014). Alzheimer's Disease Facts and Figures (USA: Alzheimer's Association). Available at: https:// www.alz.org/downloads/facts_figures_2014.pdf.

Ancoli-Israel, S., Palmer, B.W., Cooke, J.R., Corey-Bloom, J., Fiorentino, L., Natarajan, L., Liu, L., Ayalon, L., He, F., and Loredo, J.S. (2008). Cognitive effects of treating obstructive sleep apnea in Alzheimer's disease: a randomized controlled study. J. Am. Geriatr. Soc. 56, 2076-2081.

Anderson, L. (2007). Intimacy found by Alzheimer's patients. Edmonton J. 1-2. Retrieved from: https://search-proquest-com. ezproxy.uws.edu.au/docview/253458550?accountid=36155.

Anderson, S. and Brownlie, J. (2011). Build it and they will come? Understanding public views of 'emotions talk' and the talking therapies. Br. J. Guid. Counc. 39, 53-66.

Archer, N., Brown, R.G., Reeves, S., Nicholas, H., Boothby, H., and Lovestone, S. (2009). Midlife neuroticism and the age of onset of Alzheimer's disease. Psychol. Med. 39, 665-673.

Barnes, D.E. and Yeffe, K. (2011). The projected effect of risk factor reduction on Alzheimer's disease prevalence. Lancet Neurol. $10,819-828$.

Benoit, M., Berrut, G., Doussaint, J., Bakchine, S., Bonin-Guillaume, S., Fremont, P., Gallarda, T., Krolak-Salmon, P., Marquet, T., Mékiès, C., et al. (2012). Apathy and depression in mild Alzheimer's disease: a cross-sectional study using diagnostic criteria. J Alzheimers Dis. 31, 325-334.

Bialystok, E. and Luk, G. (2012). Receptive vocabulary differences in monolingual and bilingual adults. Biling. Lang. Cogn. 15, 397-401.

Bierman, E.J.M., Comijs, H.C., Jonker, C., and Beekman, A.T.F. (2007). Symptoms of anxiety and depression in the course of cognitive decline. Dement. Geriatr. Cogn. Disord. 24, 213-219.

Boyle, L.L., Lyness, J.M, Duberstein, P.R., Karuza, J., King, D.A., Messing, S., and Tu, X. (2010). Trait neuroticism, depression, and cognitive function in older primary care patients. Am. J. Geriatr. Psychiatry 18, 305-312.

Buchanan, T.W., al'Absi, M., and Lovallo, W.R. (1999). Cortisol fluctuates with increases and decreases in negative affect. ISPNE 24, 227-241.

Buchman, A.S., Boyle, P.A., Yu, L., Shah, R.C., Wilson, R.S., and Bennett, D.A. (2012). Total daily physical activity and the risk of AD and cognitive decline in older adults. Neurology 78, 1323-1329.
Cardoso, B.R., Apolinário, D., Bandeira, V.S., Busse, A.L., Magaldi, R.M., Jacob-Filho, W., and Cozzolino, S.M.F. (2016). Effects of Brazil nut consumption on selenium status and cognitive performance in older adults with mild cognitive impairment: a randomized controlled pilot trial. Eur. J. Nutr. 55, 107-116.

Chapman, B., Duberstein, P., Tindle, H.A., Sink, K.M., Robbins, J., Tancredi, D.J., and Franks, P. (2012). Personality predicts cognitive function over seven years in older persons. Am. J. Geriatr. Psychiatry 20, 612-621.

Chen, R.C.Y., Chang, Y.H., Lee, M.S., and Wahlqvist, M.L. (2011). Dietary quality may enhance survival related to cognitive impairment in Taiwanese elderly. Food Nutr. Res. 55, 1-10.

Cherbuin, N., Kim, S., and Anstey, K.J. (2015). Dementia risk estimates associated with measures of depression: a systematic review and meta-analysis. BMJ Open 5, 1-13.

Chu, H., Yang, C.Y., Lin, Y., Ou, K.L., Lee, T.Y., O’Brien, A.P., and Chou, K.R. (2014). The Impact of group music therapy on depression and cognition in elderly persons with dementia: a randomized controlled study. Biol. Res. Nurs. 16, 209-217.

Davis, D.S. (2014). Alzheimer disease and pre-emptive suicide. J. Med. Ethics. 40, 543-549.

Erickson, K.I., Voss, M.W., Prakash, R.S., Basak, C., Szabo, A., Chaddock, L., Kim, J.S., Heo, S., Alves, H., White, S.M., et al. (2011). Exercise training increases size of hippocampus and improves memory. Proc. Natl. Acad. Sci. USA 108, 3017-3022.

Farmer, A., Redman, K., Harris, T., Mahmood, A., Sadler, S., Pickering, A., and McGuffin, P. (2002). Neuroticism, extraversion, life events and depression. Br. J. Psychiatry 181, 118-122.

Fuhrer, R., Dufouil, C., and Dartigues, J.F. (2003). Exploring sex differences in the relationship between depressive symptoms and dementia incidence: prospective results from the PAQUID Study. J. Am. Geriatr. Soc. 51, 1055-1063.

Gagnon, J.F., Bertrand, J.A., and Marchand, D.G. (2012). Cognition in rapid eye movement sleep behavior disorder. Front. Neurol. 3, 1-5.

Gurvits, T.V., Shenton, M.E., Hokama, H., Ohta, H., Lasko, N.B., Gilbertson, M.W., Orr, S.P., Kikinis, R., Jolesz, F.A., McCarley, R.W., et al. (1996). Magnetic resonance imaging study of hippocampal volume in chronic, combat-related posttraumatic stress disorder. Biol. Psychiatry 40, 1091-1099.

Holwerda, T.J., Tilburg, T.G.V., Deeg, D.J.H., Schutter, N., Van, R., Dekker, J., Stek, M.L., Beekman, A.T., and Schoevers, R.A. (2016). Impact of loneliness and depression on mortality: results from the Longitudinal Ageing Study Amsterdam. BJPsych. 209, 127-134.

Horning, S.M., Melrose, R., and Sultzer, D. (2014). Insight in Alzheimer's disease and its relation to psychiatric and behavioral disturbances. Int. J. Geriatr. Psychiatry 29, 77-84.

Huang, T.L., Zandi, P.P., Tucker, K.L., Fitzpatrick, A.L., Kuller, L.H., Fried, L.P., Burke, G.L., and Carlson, M.C. (2005). Benefits of fatty fish on dementia risk are stronger for those without APOE epsilon4. Neurology 65, 1409-1414.

Hurt, C.S., Banerjee, S., Tunnard, C., Whitehead, D.L., Tsolaki, M., Mecocci, P., Kloszewska, I., Soininen, H., Vellas, B., Lovestone, S., et al. (2010). Insight, cognition and quality of life in Alzheimer's disease. J. Neurol. Neurosurg. Psychiatry 81, 331-336.

Joseph, J.A., Shukitt-Hale, B., and Willis, L.M. (2009). Grape juice, berries, and walnuts affect brain aging and behavior. J. Nutr. 139, 1813-1817.

Katz, M.J., Derby, C.A., Wang, C., Sliwinski, M.J., Ezzati, A., Zimmerman, M.E., Zwerling, J.L., and Lipton, R.B. (2016). Influ- 
ence of perceived stress on indicant amnestic mild cognitive impairment: results from the Einstein Aging Study. Alzheimer Dis. Assoc. Disord. 30, 93-98.

Kuriyama, S., Hozawa, A., Ohmori, K., Shimazu, T., Matsui, T., Ebihara, S., Awata, S., Nagatomi, R., Arai, H., and Tsuji, I. (2006). Green tea consumption and cognitive function: a crosssectional study from the Tsurugaya Project. Am. J. Clin. Nutr. 83, 355-361.

Larson, E.B. (2008). Physical activity for older adults at risk for Alzheimer disease. J Am Med Assoc 300, 1077-1079.

Liu, R., Sui, X., Laditka, J.N., Church, T.S., Colabianchi, N., Hussey, J., and Blair, S.N. (2011). Cardiorespiratory fitness as a predictor of dementia mortality in men and women. ACSM 44, 253-259.

Marx, J. (2005). Preventing Alzheimer's: a lifelong commitment? Science 309, 864-866.

McEwen, B.S. (2000). The neurobiology of stress: from serendipity to clinical relevance. Brain Res. 886, 172-189.

Mishra, S. and Palanivelu, K. (2008). The effect of curcumin (turmeric) on Alzheimer's disease: an overview. Ann. Indian Acad. Neurol. 11, 13-19.

Muthaiyah, B., Essa, M.M., Chauhan, V., and Chauhan, A. (2011). Protective effects of walnut extract against amyloid $\beta$ peptide-induced cell death and oxidative stress in PC12 cells. Neurochem. Res. 36, 2096-2103.

National Institute on Aging. (2015). Alzheimer's Disease Fact Sheet. $1-8$.

Newbery, G. (2012). Dispositional perspectives: Allport's trait theory; Eysenck's biological typology. Knowing You, Knowing Me: An Integrated View of Personality (Australia: McGraw-Hill Australia Pty Ltd), pp. 93-104.

Ng, T.P., Feng, L., Niti, M., Kua, E.H., and Yap, K.B. (2008). Tea consumption and cognitive impairment and decline in older Chinese adults. Am. J. Clin. Nutr. 88, 224-231.

Nima, A.A., Rosenberg, P., Archer, T., and Garcia, D. (2013). Anxiety, affect, self-esteem, and stress: mediation and moderation effects on depression. PLoS One 8, 1-8.

Nooyens, A.C.J., Bueno-de-Mesquita, H.B., van Boxtel, M.P.J., van Gelder, B.M., Verhagen, H., and Verschuren, W.M.M. (2011). Fruit and vegetable intake and cognitive decline in middle-aged men and women: the Doetinchem Cohort Study. Br. J. Nutr. 106, 752-761.

Nurk, E., Drevon, C.A., Refsum, H., Solvoll, K., Vollset, S.E., Nygård, O., Nygaard. H.A., Engedal, K., Tell, G.S., and Smith, A.D. (2007). Cognitive performance among the elderly and dietary fish intake: the Hordaland Health Study. Am. J. Clin. Nutr. 86,1470-1478.

O’Connor, E.M., Power, S.E., Fitzgerald, G.F., and O'Toole, P.W. (2012). Fish-oil consumption is inversely correlated with depression and cognition decline in healthy Irish elderly adults. Proc. Nutr. Soc. 71, 1.

O’Luanaigh, C., O'Connell, H., Chin, A.-V., Hamilton, F., Coen, R., Walsh, C., Walsh, J.B., Caokley, D., Cunningham, C., and Lawlor, B.A. (2012). Loneliness and cognition in older people: The Dublin Healthy Ageing study. Aging Mental Health 16, 347-352.

Ohayon, M.M. and Vecchierini, M.F. (2005). Normative sleep data, cognitive function and daily living activities in older adults in the community. Sleep 28, 981-989.

Osorio, R.S., Pirraglia, E., Agüera-Ortiz, L.F., During, E.H., Sacks, H., Ayappa, I., Walsleben, J., Mooney, A., Hussain, A., Glodzik, L., et al. (2011). Greater risk of Alzheimer's disease in older adults with insomnia. J. Am. Geriatr. Soc. 59, 559-562.

Park, S.K., Jung, I.C., Lee, W.K., Lee, Y.S., Park, H.K., Go, H.J., Kim, K., Lim, N.K., Hong, J.T., Ly, S.Y., et al. (2011). A combination of green tea extract and L-theanine improves memory and attention in subjects with mild cognitive impairment: a double-blind placebo-controlled study. J. Med. Food 14, 334-343.

Pe'neau, S., Galan, P., Jeandel, C., Ferry, M., Andreeva, V., Hercberg, S., Kesse-Guyot, E., and the SU.VI.MAX 2 Research Group. (2011). Fruit and vegetable intake and cognitive function in the SU.VI.MAX 2 prospective study. Am. J. Clin. Nutr. 94, 1295-1303.

Pereira, A.C., Huddleston, D.E., Brickman, A.M., Sosunov, A.A., Hen, R., McKhann, G.M., Sloan, R., Gage, F.H., Brown, T.R., and Small, S.A. (2007). An in vivo correlate of exercise-induced neurogenesis in the adult dentate gyrus. Proc Natl Acad Sci USA 104, 5638-5643.

Poulin, S.P., Dautoff, R., Morris, J.C., Barrett, L.F., and Dickerson, B.C. (2011). Amygdala atrophy is prominent in early Alzheimer's disease and relates to symptom severity. Psychiatry Res. 194, 7-13.

Poulose, S.M., Miller, M.G., and Shukitt-Hale, B. (2014). Role of walnuts in maintaining brain health with age. J. Nutr. 144, 561-566.

Qin, B., Plassman, B.L., Edwards, L.J., Popkin, B.M., Adair, L.S., and Mendez, M.A. (2014). Fish intake is associated with slower cognitive decline in Chinese older adults. J. Nutr. 144, 1579-1585.

Rest, O.V., Zwaluw, N.L.V., and Groot, L.C.P.G.M. (2013). Literature review on the role of dietary protein and amino acids in cognitive functioning and cognitive decline. Amino Acids 45, 1035-1045.

Sapolsky, R.M. (1996). Why stress is bad for your brain. Science 273, 749-750.

Sheline, Y.I., Gado, M.H., and Kraemer, H.C. (2003). Untreated depression and hippocampal volume loss. Am. J. Psychiatry 160, 1516-1518.

Spira, A.P., Chen-Edinboro, L.P., Wu, M.N., and Yaffe, K. (2014). Impact of sleep on the risk of cognitive decline and dementia. Curr. Opin. Psychiatry 27, 478-483.

Starkstein, S.E., Jorge, R., Mizrahi, R., Adrian, J., and Robinson, R.G. (2007). Insight and danger in Alzheimer's disease. Eur. J. Neurol. 14, 455-460.

Stranks, E.K. and Crowe, S.F. (2016). The cognitive effects of obstructive sleep apnea: an updated meta-analysis. Arch. Clin. Neuropsychol. 31, 186-193.

Sullivan, M.G. (2008). Fitness may limit brain atrophy in Alzheimer's: the level of fitness was strongly related to volume in the parietal area and also in the hippocampus. CPSN 36, 1-2.

Tanday, S. (2007). Behind the headlines: is loneliness linked to Alzheimer's? GP 13. Retrieved from: https://search-proquest-com. ezproxy.uws.edu.au/docview/225161564? accountid=36155.

Tortora, G. and Derrickson, B. (2006). The Brain and Cranial Nerves. Principles of Anatomy and Physiology. B. Roesch, K. Trost, eds. 11th ed. (United States: John Wiley and Sons, Inc.), pp. 473-523.

Tunnard, C., Whitehead, D., Hurt, C., Wahlund, L.O., Mecocci, P., Tsolaki, M., Vellas, B., Spenger, C., Kłoszewska, I., Soininen, H., et al. (2011). Apathy and cortical atrophy in Alzheimer's disease. Int. J. Geriatr. Psychiatry 26, 741-748.

University of lowa. (2010). Health science; study: patients with amnesia still feel emotions, despite memory loss. Pain and Central Nervous System Week 61. 
Van Cauter, E., Leproult, R., and Plat, L. (2000). Age-related changes in slow wave sleep and REM sleep and relationship with growth hormone and cortisol levels in healthy men. J Am Med Assoc 284, 861-868.

Willis, L.M., Shukitt-Hale, B., Cheng, V., and Joseph, J.A. (2009). Dose-dependent effects of walnuts on motor and cognitive function in aged rats. Br. J. Nutr. 101, 1140-1144.

Wilson, R.S., Bennett, D.A., Mendes de Leon, C.F., Bienias, J.L., Morris, M.C., and Evans, D.A. (2005). Distress proneness and cognitive decline in a population of older persons. PNEC 30, 11-17.

Wilson, R.S., Schneider, J.A., Boyle, P.A., Arnold, S.E., Tang, Y., and Bennett, D.A. (2007a). Chronic distress and incidence of mild cognitive impairment. Neurology 68, 2085-2092.

Wilson, R.S., Krueger, K.R., Arnold, S.E., Schneider, J.A., Jeremiah, F.K., Barnes, L.L., Tang, Y., and Bennett, D.A. (2007b). Loneliness and Risk of Alzheimer Disease. Arch. Gen. Psychiatry 64, 234-240.

Wilson, R.S., Begeny, C.T., Boyle, P.A., Schneider, J.A., and Bennett, D.A. (2011). Vulnerability to stress, anxiety, and development of dementia in old age. Am. J. Geriatr. Psychiatry 19, 327-334.
Xu, Y., Lin, D., Li, S., Li, G., Shyamala, S.G., Barish, P.A., Vernon, M.M., Pan, J., and Ogle, W.O. (2009). Curcumin reverses impaired cognition and neuronal plasticity induced by chronic stress. Neuropharmacology 57, 463-471.

Yaffe, K., Blackwell, T., Gore, R., Sands, L., Reus, V., and Browner, W.S. (1999). Depressive symptoms and cognitive decline in nondemented elderly women: a prospective study. Arch. Gen. Psychiatry 56, 425-430.

Yaffe, K., Vittinghoff, E., Lindquist, K., Barnes, D., Covinsky, K.E., Neylan, T., Kluse, M., and Marmar, C. (2010). Post-traumatic stress disorder and risk of dementia among U.S. veterans. Arch. Gen. Psychiatry 67, 608-613.

Yehuda, S., Rabinovitz, S., Carasso, R.L., and Mostofsky, D.I. (2002). The role of polyunsaturated fatty acids in restoring the aging neuronal membrane. Neurobiol. Aging 23, 843-853.

Zhang, Y., Chen, J., Qiu, J., Li, Y., Wang, J., and Jiao, J. (2016). Intakes of fish and polyunsaturated fatty acids and mild-to-severe cognitive impairment risks: a dose-response meta-analysis of 21 cohort studies. Am. J. Clin. Nutr. 103, 330-340. 